\title{
MicroRNA-203 inhibits the progression of esophageal squamous cell carcinoma with restored epithelial tissue architecture in vivo
}

\author{
TOMOYUKI OKUMURA $^{1}$, YUTAKA SHIMADA ${ }^{1,2}$, MAKOTO MORIYAMA $^{1}$, \\ YOSHINORI TAKEI $^{2}$, TETSUYA OMURA ${ }^{1}$, SHINICHI SEKINE ${ }^{1}$, TAKUYA NAGATA ${ }^{1}$, \\ KAZUHARU SHIMIZU ${ }^{2}$ and KAZUHIRO TSUKADA ${ }^{1}$ \\ ${ }^{1}$ Department of Surgery and Science, Graduate School of Medicine and Pharmaceutical Sciences, \\ University of Toyama, Toyama 930-0194; ${ }^{2}$ Department of Nanobio Drug Discovery, Graduate School \\ of Pharmaceutical Sciences, Kyoto University, Sakyo-Ku, Kyoto 606-8501, Japan
}

Received January 18, 2014; Accepted March 11, 2014

DOI: $10.3892 /$ ijo.2014.2365

\begin{abstract}
MicroRNA (miR)-203 has been shown to induce squamous differentiation of epidermal stem cells through the suppression of $\mathrm{p} 63$. The aim of this study was to assess the tumor suppressor effect of miR-203 in esophageal squamous cell carcinoma (ESCC) with focus on the regulation of the cell fate decisions and organization of tumor tissue architecture in vivo. Our investigation establishing stable clones from ESCC cell lines with induced miR-203 expression resulted in significant growth inhibition in a mouse xenograft model. Small foci were observed in xenograft tumors with stratified squamous differentiation in conjunction with restored baso-apical polarity. The expression of the basement membrane protein laminine was localized at the center of the foci and the basal cell marker p75NTR was expressed in the innermost layer. The expression of ki67 and p63 was co-localized at the center layers, while involucrin was expressed in the outer layers. Flow cytometry revealed that the p75NTR-positive cells expressing p63 and Bmil were well maintained, while the expression of p63 was suppressed in the p75NTR-negative cells. Our cDNA microarray analysis demonstrated the upregulation of genes involved in regulating tissue architecture, such as BMP-4 and ZO-1 in the mir-203 transfectant. Investigation using surgically removed ESCC specimens revealed that the expression of miR-203 significantly correlated with a favorable prognosis. These results demonstrated that miR-203 regulated both basal and supra-basal cell components to induce differentiation with restored epithelial tissue architecture, leading to significant tumor growth inhibition in vivo. Those results suggest the
\end{abstract}

Correspondence to: Dr Tomoyuki Okumura, Department of Surgery and Science, Graduate School of Medicine and Pharmaceutical Sciences, University of Toyama, Sugitani 2630, Toyama 930-0194, Japan

E-mail: okumura@med.u-toyama.ac.jp

Key words: esophageal cancer, miR-203, differentiation, basal cells, tissue architecture use of miR-203 as a novel therapeutic and diagnostic target in patients with ESCC.

\section{Introduction}

Despite advances in its diagnosis and multimodal therapies, the prognosis for patients with esophageal squamous cell carcinoma (ESCC) remains poor (1). Therefore, innovative strategies derived from a better understanding of the biological basis of ESCC are needed to further improve the outcome of patients with this disease.

Squamous epithelia, such as the epidermis and esophageal epithelium, consist of heterogeneous cell populations $(2,3)$. Stem cells residing at the basal layer possess self-renewal ability, which maintains the stem cell pool and also gives rise to actively proliferating cells. The proliferating cells move from the basal layer to the supra-basal layers and then exit the cell cycle to proceed terminal differentiation (2). On the other hand, malignant tumors lose original tissue organization with disrupted cell fate regulation and the extent of the disorder has been associated with poor clinical outcomes (3). Intratumor heterogeneity and a small number of cells with stem cell properties in cancer and its cell fate regulation have been demonstrated in tumors from several cancers, including skin, and breast cancer $(4,5)$. However, the molecular mechanisms involved in balancing stem cell self-renewal and differentiation in ESCC have not yet been elucidated.

MicroRNAs (miRNAs) are small, single-stranded, noncoding RNAs that play a key role in development and various biological processes through the post-transcriptional regulation of gene expression (6). The expression of miR-203 has been reported in suprabasal cells in normal skin and promotes epidermal differentiation by regulating proliferation through the suppression of p63 (7,8). A study using a transgenic mouse model demonstrated a depletion of epidermal basal cells and reduced epidermal thickness due to the induction of miR-203 (8). To date, in vitro tumor suppressor effect of mir-203 in various cancer have been reported, however, the role of miR-203 in the cell fate regulation of specific cell compartment, differentiation, and in vivo tissue organization in malignant tumors has not yet been elucidated. 
In this study, to further assess the use of miR-203 as a novel therapeutic target for ESCC, we first examined the tumor suppressor effect of miR-203, with focus on regulation of the cell fate decision and differentiation in vitro and in vivo. We then examined the expression of miR-203 in surgically removed ESCC specimens to assess its clinicopathological significance.

\section{Materials and methods}

Cell culture. Human esophageal squamous carcinoma cell lines (KYSE520 and 790) were established by Shimada and colleagues, and cultured in Ham's F12/RPMI-1640 with 2\% FCS according to a previously reported method $(9,10)$.

miR-203 expressing vector and stable transfection. cDNAs encoding Hsa-miR-203 sequences were subcloned into a pBApro-CMV Neo DNA plasmid (Takara Bio Inc., Shiga, Japan) in which a neomycin selectable marker was encoded. The hsa-miR-203 plasmid or mock plasmid was transfected into KYSEs with lipofectamine 2000 (Invitrogen, Carlsbad, CA, USA) according to a standard protocol (Lipofectamine $10 \mu \mathrm{l}$ per vector DNA $4 \mu \mathrm{g}$ ). Cells were then cultured with medium containing $400 \mu \mathrm{g} / \mathrm{ml}$ of G418. Colonies were chosen 7 days after transfection and were then cultured.

Flow cytometry and cell sorting. Adherent cells were trypsinized, washed once in cold PBS, and $2 \times 10^{5}$ viable cells were resuspended in $50 \mu \mathrm{l}$ of PBS with $0.5 \%$ bovine serum albumin (staining buffer). Cells were incubated with $1 \mathrm{mg} / \mathrm{ml}$ of mouse anti-p75NTR (clone NGFR5) for single staining before fluorescence-activated cell sorting analysis (Becton Dickinson, Inc., San Jose, CA, USA). After washing with staining buffer, cells were resuspended in $50 \mu \mathrm{l}$ of staining buffer and incubated with $1 \mathrm{mg} / \mathrm{ml}$ of the FITC-conjugated goat anti-mouse immunoglobulin $\mathrm{G}(\mathrm{IgG})$ antibody. Both primary and secondary staining reactions were carried out for $15 \mathrm{~min}$ at room temperature. Non-specific isotype matched antibodies were used as controls. DAPI was added to exclude dead cells. Cells were analyzed using BD LSRII (Becton Dickinson) and results were analyzed with Cell Quest software (Becton Dickinson).

RNA extraction from cultured cells and tumors obtained from the xenograft model. Total RNA was extracted from cells using the TRIzol Reagent (Invitrogen) method. Murine tissue (0.5-2 mg) was homogenized using a bench-top homogenizer in $1 \mathrm{ml} \mathrm{TRIzol} \mathrm{Reagent} \mathrm{(Invitrogen).}$

$q R T$-PCR analysis for $m R N A$. cDNA was synthesized using the Superscript First-Strand Synthesis System (Invitrogen). Realtime PCR reactions with the QuantiTect SYBR green PCR kit (Qiagen, Maryland, MA, USA) were run on an ABI Prism ${ }^{\circledR}$ 7300 (Applied Biosystems, Branchburg, NJ, USA). mRNA quantities were analyzed in duplicate and normalized against GAPDH as an internal control. Results were expressed as relative gene expression using the $\Delta \Delta \mathrm{Ct}$ method. The following primer sequences were used: Involucrin: forward - tcctcctcca gtcaataccc, reverse - gctgatccctttgtgtt; p63: forward - cagacttg ccaaatcatcc, reverse - cagcattgtcagtttcttagc; Bmi1: forward ggagaccagcaagtattgtccttttg, reverse - cattgctgctgggcatcgtaag. $q R T-P C R$ analysis for $m i R-203$. cDNA was prepared from miRNA samples using the Taq Man microRNA reverse transcription kit on the ABI Prism 7000 real-time PCR system according to the manufacturer's instructions (Applied Biosystems). Predesigned Taq Man microRNA assays for hsa-miR-203 (Assay ID 000507) was purchased from Applied Biosystems. qRT-PCR was performed using a Taq Man universal PCR master mix, according to the manufacturer's protocol (Applied Biosystems).

Mouse xenograft model. All mouse studies were carried out under the approval of the Institutional Review Board of the University of Toyama (no. S-2008MED-56). KYSE cells $\left(1 \times 10^{6}\right)$ in $100 \mu \mathrm{l}$ PBS were injected subcutaneously into the right flank of four week-old female CAnN.Cg-Foxn1nu/CrlCrlj (BALB/c) nude mice (Charles River Laboratories, Yokohama, Japan). Animals were sacrificed at week 8 and tumor tissues were harvested. Parts of the tumor tissues were used for RNA and the rest were fixed in $10 \%$ formalin and embedded in paraffin.

Histopathology and immunohistochemistry. Sections (4 $\mu \mathrm{m})$ were cut from paraffin blocks, deparaffinized with xylene, and rehydrated through a graded alcohol series. These sections were then autoclaved at $121^{\circ} \mathrm{C}$ in Target Retrieval Solution (Dako Cytomation, Kyoto, Japan) for $5 \mathrm{~min}$ and cooled to room temperature to unmask antigens. Immunostaining was performed with Envision Plus kits/horseradish peroxidase/3,3'diaminobenzidine (Dako Cytomation) according to the manufacturer's instructions. Primary antibodies were incubated at room temperature for $1 \mathrm{~h}$, or at $4^{\circ} \mathrm{C}$ overnight. Slides were counterstained with Mayer's hematoxylin. The primary antibodies used in this study were as follows: rabbit polyclonal anti-Ki67, clone ab66155 (Abcam Inc., Cambridge, MA, USA) dilution1:200, mouse monoclonal anti-involucrin, clone SY5 (Santa Cruz Biotechnology, Inc., CA, USA) dilution 1:50, mouse monoclonal anti-p63, clone 4A4 (Abcam Inc.) dilution 1:1, mouse monoclonal anti-Bmi1, clone 1.T.21 (Abcam Inc.) dilution 1:100, mouse monoclonal anti-p75NTR, clone NGFR5 (Santa Cruz Biotechnology, Inc.) dilution 1:50, and rabbit polyclonal anti-Laminin (Abcam Inc.) dilution 1:100.

cDNA microarray. A 3D-Gene Human Oligo chip 25 k (Toray Industries, Tokyo, Japan) was used (25,370 distinct genes) according to the supplier's protocols (www.3d-gene.com).

Patients and surgical specimens. Written informed consent for this research was obtained from patients prior to surgery with approval by the Institutional Review Board (no. 20-75). From a total of 100 serial ESCC patients who had undergone esophagectomy between 1997 and 2007 in the University of Toyama Hospital, RNA was obtained with appropriate quality from the FFPE samples of 31 patients. Then patients with disease stage I or IV and/or patients who received non-radical surgery and/or patients who died within 6 months of surgery were excluded from this study. Finally, in total 20 cases were analyzed in this study. Information on gender, age, stage of disease and histopathological factors was abstracted from the medical records. All of the tumors were confirmed as ESCC by the Clinicopathological Department of the hospital. All 
A

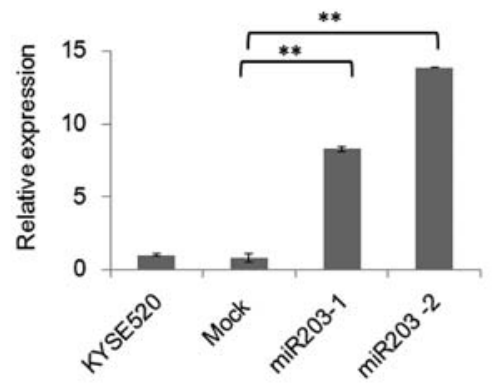

B
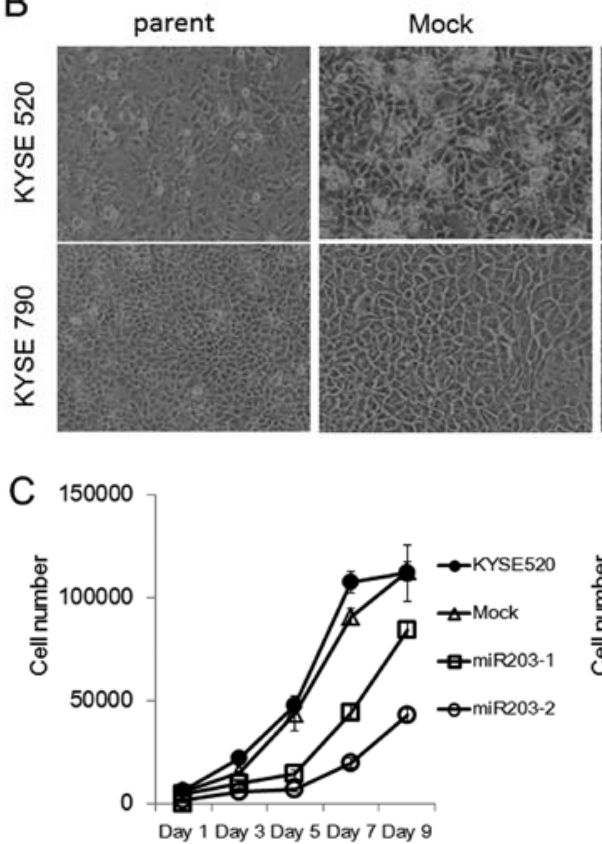

D

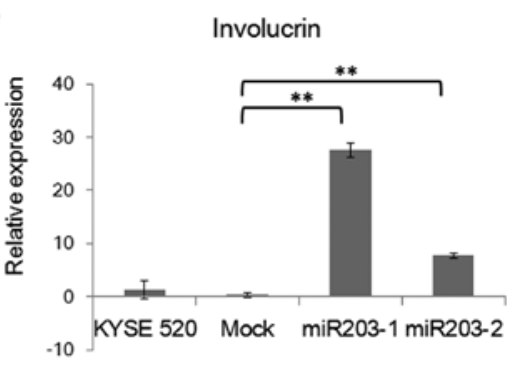

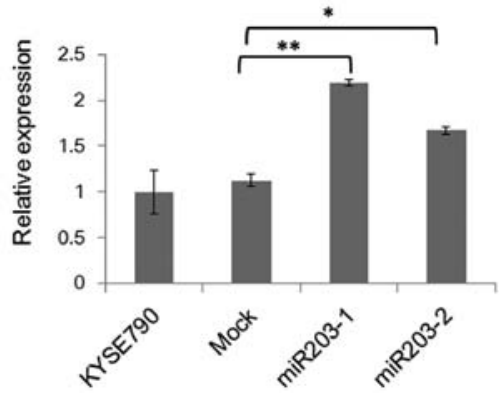

miR203-1
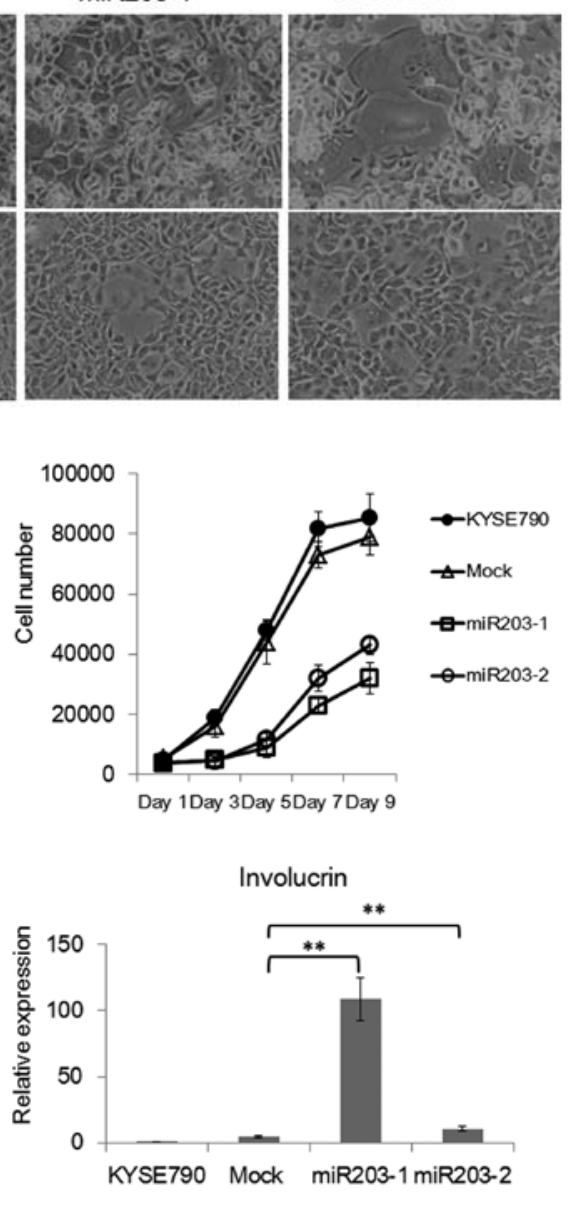

Figure 1. Establishment of miR-203 stable clones, and their growth and differentiation. miR-203 stable clones and mock transfectants were established from KYSE520 and KYSE790, namely, KYSE520 mock, KYSE520 miR203-1, KYSE520 miR203-2, KYSE790 mock, KYSE790 miR203-1 and KYSE790 miR203-2. (A) Relative expression of miR-203 in the miR-203 stable clones detected by real-time PCR (mean $\pm \mathrm{SD},{ }^{,} \mathrm{P}<0.05$, $\left.{ }^{* *} \mathrm{P}<0.01\right)$. (B) Representative phase contrast image of the miR-203 stable clones. (C) Growth curves of the miR-203 stable clones ( $n=3$, mean \pm SD). (D) Relative expression of involucrin in the miR-203 stable clones detected by real-time PCR (mean $\left.\pm \mathrm{SD},{ }^{*} \mathrm{P}<0.05,{ }^{* * *} \mathrm{P}<0.01\right)$.

cases were classified according to the International Union Against Cancer TNM Classification 7th edition (11).

RNA extraction from FFPE specimens. Sections $(10 \mu \mathrm{m})$ were prepared from each FFPE specimen. Paraffin was removed by treatment with xylene and tissues were washed with ethanol twice to remove xylene. Tissues were then treated with proteinase $\mathrm{K}$ at $37^{\circ} \mathrm{C}$ overnight. Following centrifugation, the supernatant was processed with a silicabased spin column (Toray Industries) in order to obtain purified total RNA. The degrees of RNA cross-linking and RNA degradation were analyzed by electrophoresis using an Agilent 2100 Bioanalyzer (Agilent Technologies, Santa Clara, CA, USA).
miRNA assays. miRNA profiling was examined using a Toray 3D-Gene ${ }^{\circledR}$ miRNA oligo chip (Toray Industries) on which 885 genes were mounted. The detailed procedure of the experiment has been previously described (12). We defined tumors with high miR-203 expression when the relative expression level of miR-203 was 2-fold higher than that in normal counterparts from the same resected specimen.

Statistical analysis. mRNA expression levels in mouse tumor and cultured cells were analyzed by the t-test. The Kaplan-Meier method was used to estimate patient survival. Differences in survival for the overexpression ( $\mathrm{T} / \mathrm{N}$ ratio $>2.0$ ) of miR-203 were analyzed using the log-rank test. $\mathrm{P}<0.05$ was set as significance. 


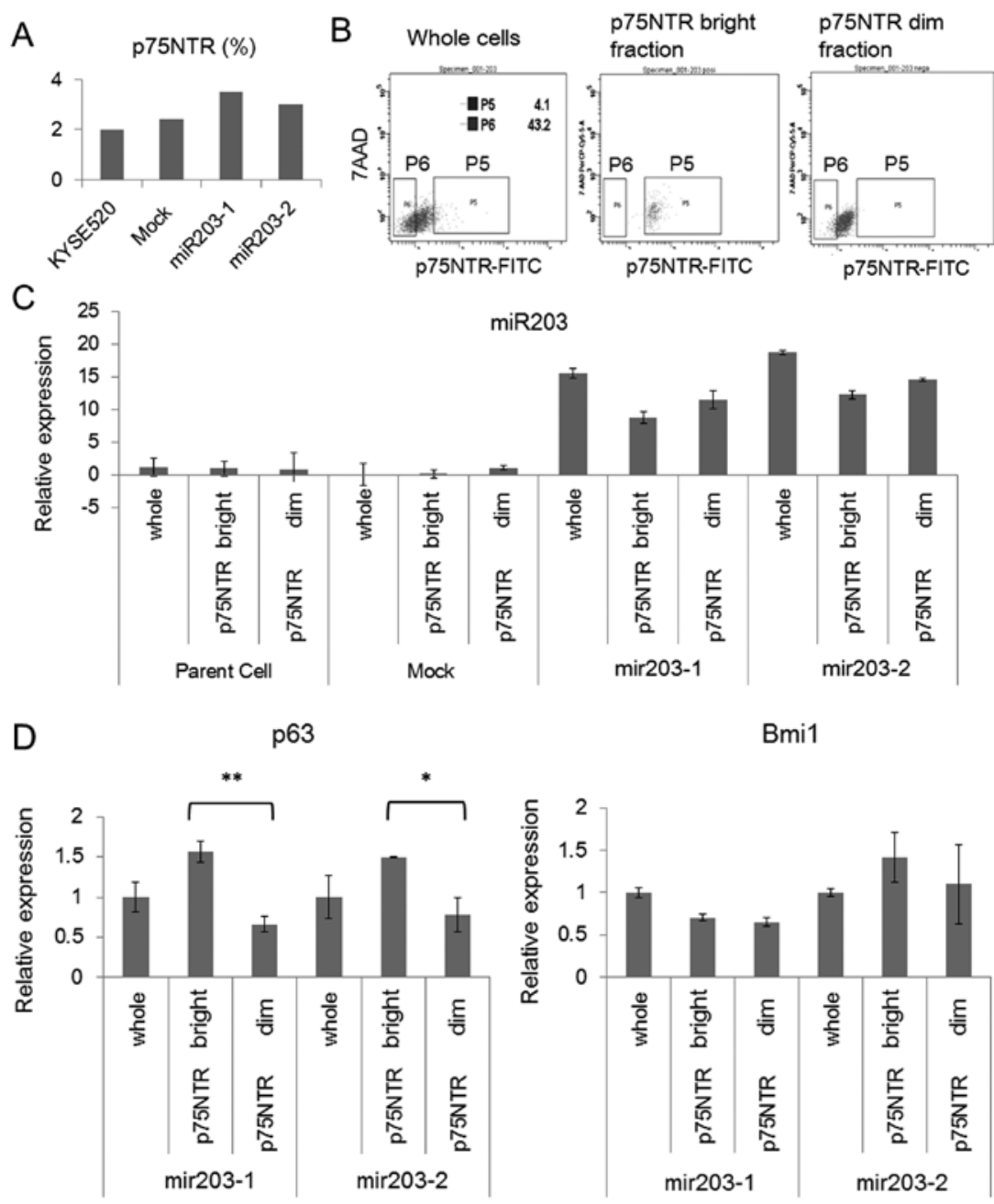

Figure 2. The expression of 'stemness' genes in miR-203 stable clones. (A) The proportion of cells strongly expressing p75NTR in KYSE520, KYSE520 mock, KYSE520 miR203-1, KYSE520 miR203-2 detected by flow cytometry. (B) Fractionation of KYSE520 miR203-2 into p75NTR bright and dim cells by a cell sorter. (C) Relative expression of miR-203 in fractionated cells from KYSE520, KYSE520 mock, KYSE520 miR203-1 and KYSE520 miR203-2 detected by real-time PCR (mean \pm SD). (D) Relative expression of p63 and Bmi-1 in fractionated cells from KYSE520 miR203-1 and KYSE520 miR203-2 detected by real-time PCR (mean $\left.\pm \mathrm{SD},{ }^{*} \mathrm{P}<0.05,{ }^{* *} \mathrm{P}<0.01\right)$.

\section{Results}

Establishment of miR-203 stable clones, their growth and differentiation. To investigate the functional role of miR-203 in ESCC, we established stable clones constitutively expressing miR-203 by transfecting the miR-203 plasmid into two ESCC cell lines (KYSE520 and KYSE790). We established a mock transfectant and 2 stable transfectants each from KYSE520 and KYSE790, namely, KYSE520 mock, KYSE520 miR203-1, KYSE520 miR203-2, KYSE790 mock, KYSE790 miR203-1 and KYSE790 miR203-2. miR-203 expression levels in miR203 transfectants were 7-14-fold higher than those in the parent cell and mock transfectant established from KYSE520 (Fig. 1A), whereas, miR-203 expression levels in KYSE790 miR203 transfectants were 1.5-2.1-fold higher than those in the parent cell and mock transfectant (Fig. 1A). Morphologically, the parent cell and the mock transfectant had a homogeneous small angular shape, while miR-203 transfectants were heterogeneous in size and shape, with large round cells with a decreased nuclear-cytoplasmic ratio (Fig. 1B). Cell proliferation was significantly lower in miR-203 transfectants than in the parent cell and the mock transfectant
(Fig. 1C). Involucrin expression levels were 8-27-fold higher in the miR-203 transfectants than in the parent cell and mock transfectant (Fig. 1D). Taken together, the induced expression of miR-203 resulted in cell growth inhibition and terminal differentiation in ESCC cell lines.

Expression of 'stemness' genes in the miR-203 stable clones. To investigate the miR-203 induced phenotypic change in specific cell subsets in ESCC, we focused on the expression of p75NTR, which has been reported to be expressed exclusively in basal cells of normal esophageal epithelia (13). There was no significant difference among KYSE520, KYSE520-Mock and the miR-203 transfectants in the proportion of p75NTRbright cells detected by flow cytometry (Fig. 2A). We isolated the p75NTR bright/dim fraction from the parent, mock and miR-203 stable clones, respectively (Fig. 2B). In the parent and mock transfectant, the relative expression level of miR-203 in p75NTR dim cells was higher than that in p75NTR bright cells (Fig. 2C). miR-203 expression was induced in both the p75NTR bright and dim cells of the miR-203 stable clone (Fig. 2C), which indicated that miR-203 expression was broadly induced in the stable clone by the vector with the 

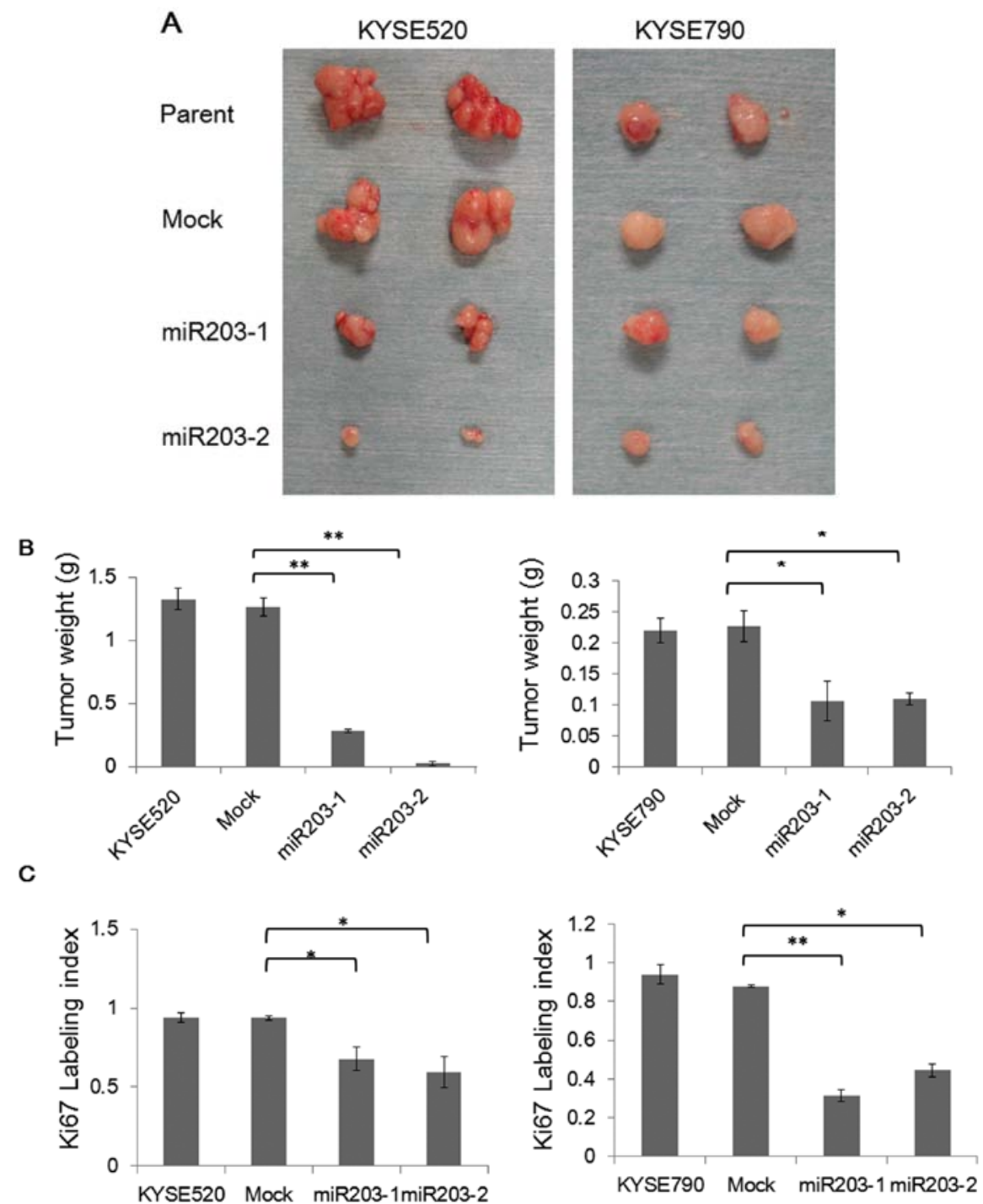

Figure 3. Tumor formation of miR-203 stable clones in the mouse xenograft model. (A) Gross appearance of the tumors established from subcutaneous injection of the miR-203 stable clones into nude mice. (B) Weight of the tumors established from the mouse xenograft $\left(\mathrm{n}=3\right.$, mean $\pm \mathrm{SD},{ }^{*} \mathrm{P}<0.05$, $\left.{ }^{* *} \mathrm{P}<0.01\right)$. $(\mathrm{C}) \mathrm{Ki} 67$ labeling index of the tumors. The percentage of Ki67-positive cells was determined by scoring 1000 cells $\left(n=3\right.$, mean $\pm S D$, $\left.{ }^{*} \mathrm{P}<0.05\right)$.

CMV promoter. Then we detected the expression of p63 and Bmi-1, which have been reported to regulate the self-renewal and differentiation of normal epithelial stem cells (14-16), by RT-PCR. In the miR-203 stable clone, p63 expression was higher in the p75NTR bright fraction than in the p75NTR dim fraction, while Bmil was expressed to the same extent in both the p75NTR bright and dim fractions (Fig. 2D). These results indicated that suppression of p63 in the p75NTR dim fraction was involved in the miR-203 induced phenotypic change in vitro.

Tumor formation of miR-203 stable clones in the mouse xenograft model. We subcutaneously injected miR-203 stable clones into mice ( $\mathrm{n}=3$ in each clone). Four weeks after the injection, tumors were established from all the cell lines including miR-203 stable clones (Fig. 3A), which indicated that the induction of miR-203 did not affect the tumor-forming efficacy of the cells. On the other hand, the size of the tumors established from injecting miR-203 stable clones was significantly smaller than that from the parent and mock transfectant
(Fig. 3B). Histological examination of H\&E-stained sections of the tumors showed small cancer cells homogeneously distributed in the tumors established from injecting the parent and mock transfectant (Fig. 3D). In contrast, multi-centric foci were seen in the tumors established from injecting miR-203 stable clones (Fig. 3D). The cells were large, eosinophilic, and polygonal, and appeared to be stratified from the center of the foci toward the outer layer in which cornification was seen, resembling the architectural pattern of squamous epithelia.

Ki67 staining revealed that proliferating cells were broadly distributed in tumors established from the parent and mock transfectant, while proliferating cells were limited to the center area of the foci in tumors from miR-203 stable clones (Fig. 3E). The ki67 labeling index was significantly lower in tumors from miR-203 stable clones than in the parent and mock transfectant (Fig. 3C). Very weak involucrin expression was observed in tumors from the parent and mock transfectant, while involucrin was expressed in cornified cells at the outer layer of the foci in tumors from miR-203 stable clones (Fig. 3F). 

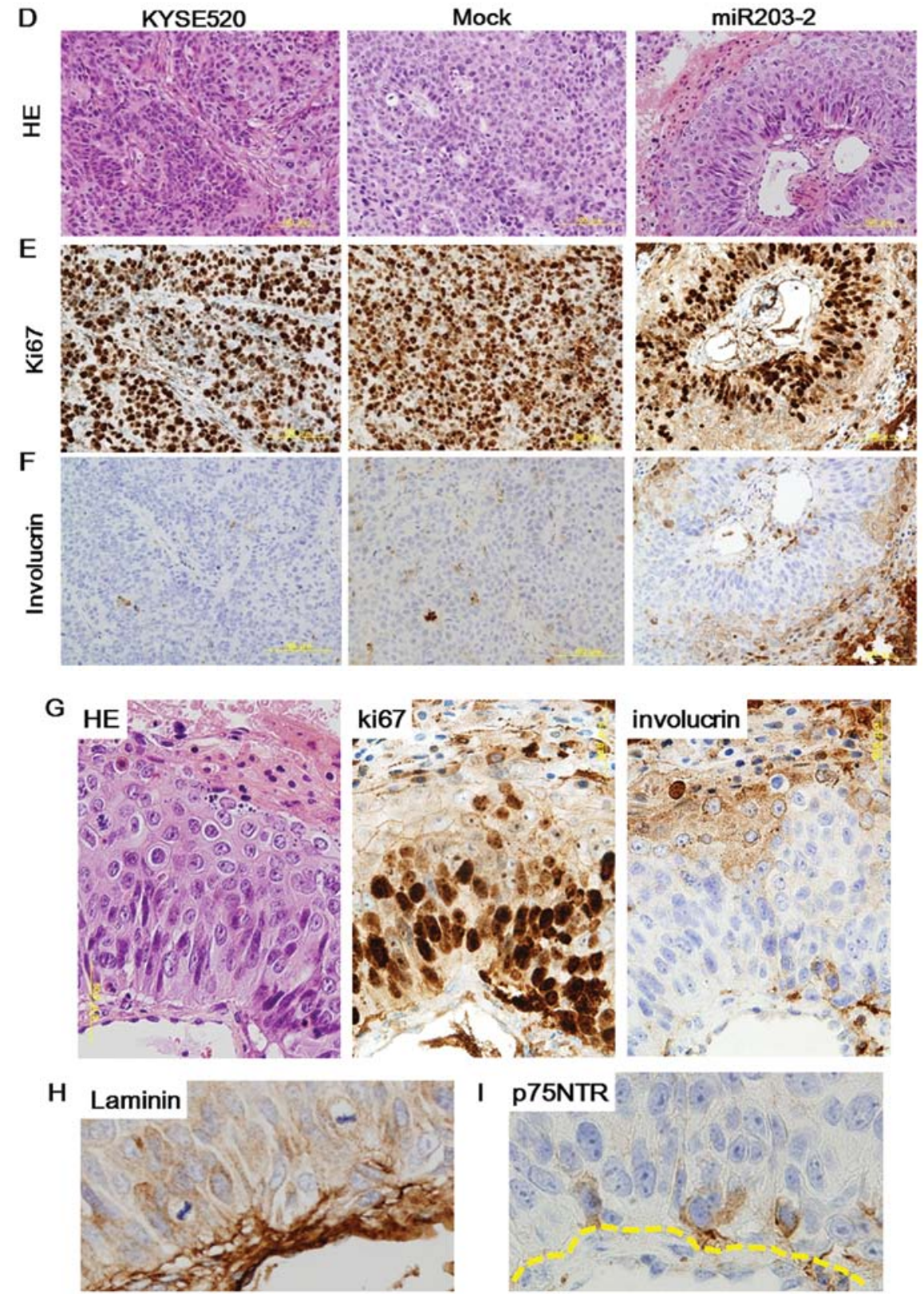

Figure 3. Continued. Tumor formation of miR-203 stable clones in the mouse xenograft model. (D) Hematoxylin and eosin (H\&E) staining in the mouse xenograft tumors established from KYSE520, KYSE520 mock and KYSE520 miR203-2. Original magnification, x200. (E) Immunohistochemical staining for ki67 in the mouse xenograft tumors. Original magnification, x200. (F) Immunohistochemical staining for Involucrin in the mouse xenograft tumors. Original magnification, x200. (G) High power view of serial sections from tumors established from a subcutaneous injection of KYSE520 miR203-2 into mice shown in (D). Original magnification, $\mathrm{x}$ 400. (H) Immunohistochemical staining for Laminin in the serial sections from tumors established from a subcutaneous injection of KYSE520 miR203-2 into mice. Original magnification, x400. (I) Immunohistochemical staining for p75NTR in the serial sections from tumors established from a subcutaneous injection of KYSE520 miR203-2 into mice. Original magnification, x400.

Detailed high power observations of serial sections of tumors from miR-203 stable clones showed that small spindleshaped cells lined the center of the foci as if to mimic cells on the basal layer in squamous epithelia. Round cells lined the middle layers, and flat cornified cells lined the outer layers (Fig. 3G). Ki67 was expressed on cells at the innermost layers (Fig. 3G), and involucrin was expressed in cornified cells at the outer layers (Fig. 3G). Laminine, a basal membrane protein, was expressed in the inner area of the innermost layer (Fig. 3H). p75NTR expression was limited to a small number of cells scattered on the innermost layer (Fig. 3I). The immunohistochemical detection on serial sections indicated recovered epithelial tissue polarity with basement membrane formation upon the induction of miR-203 in vivo.

Molecular mechanism involved in the miR-203-induced phenotypic change. To investigate the molecular mechanism involved in the miR-203-induced phenotypic change, we profiled gene expression in KYSE520 mock and KYSE520 miR203-2 using cDNA microarray analysis (TORAY-3D gene chip with 25,370 distinct genes). The upregulation of a total of 1539 genes was >2-fold higher in the miR-203 transfectant than in the mock transfectant (data not shown). Genes encoding basal membrane proteins such as laminin and collagen type 4 
Table I. The expression of genes encoding basal membrane proteins and genes involved in regulating tissue architecture in KYSE520 miR203-2 (Cy5) relative to KYSE520 mock (Cy3).

\begin{tabular}{|c|c|c|c|}
\hline Symbol & Gene name & Fold (Cy3/Cy5) & LOG2 \\
\hline LAMA1 & Laminin subunit $\alpha-1$ precursor & 0.77 & -0.39 \\
\hline LAMA2 & Laminin subunit $\alpha-2$ precursor & 2.60 & 1.38 \\
\hline LAMA3 & Laminin subunit $\alpha-3$ precursor & 4.43 & 2.15 \\
\hline LAMA5 & Laminin subunit $\alpha-5$ precursor & 1.02 & 0.03 \\
\hline LAMB1 & Laminin subunit $\beta-1$ precursor & 2.04 & 1.03 \\
\hline LAMB2 & Laminin subunit $\beta-2$ precursor & 1.19 & 0.25 \\
\hline LAMB3 & Laminin subunit $\beta-3$ precursor & 2.20 & 1.14 \\
\hline LAMB4 & Laminin subunit $\beta-4$ precursor & 0.91 & -0.13 \\
\hline LAMC1 & Laminin subunit $\gamma$-1 precursor & 2.12 & 1.08 \\
\hline LAMC2 & Laminin subunit $\gamma-2$ precursor & 1.25 & 0.32 \\
\hline LAMC3 & Laminin subunit $\gamma-3$ precursor & 0.74 & -0.44 \\
\hline COL4A3BP & Collagen type IV $\alpha$-3-binding protein & 2.37 & 1.24 \\
\hline COL4A4 & Collagen $\alpha-4(\mathrm{IV})$ chain precursor & 11.99 & 3.58 \\
\hline COL4A4 & Collagen $\alpha-4(\mathrm{IV})$ chain precursor & 2.35 & 1.23 \\
\hline COL4A5 & Collagen $\alpha-5$ (IV) chain precursor & 3.63 & 1.86 \\
\hline COL4A6 & Collagen $\alpha-6(I V)$ chain precursor & 3.78 & 1.92 \\
\hline COL8A1 & Collagen $\alpha-1$ (VIII) chain precursor & 4.44 & 2.15 \\
\hline ROCK1 & Rho-associated protein kinase 1 & 5.52 & 2.46 \\
\hline BMP4 & Bone morphogenetic protein 4 precursor & 2.96 & 1.57 \\
\hline TJP1 (ZO-1) & Tight junction protein ZO-1 & 2.04 & 1.03 \\
\hline
\end{tabular}

A KYSE520 Mock miR203 -2

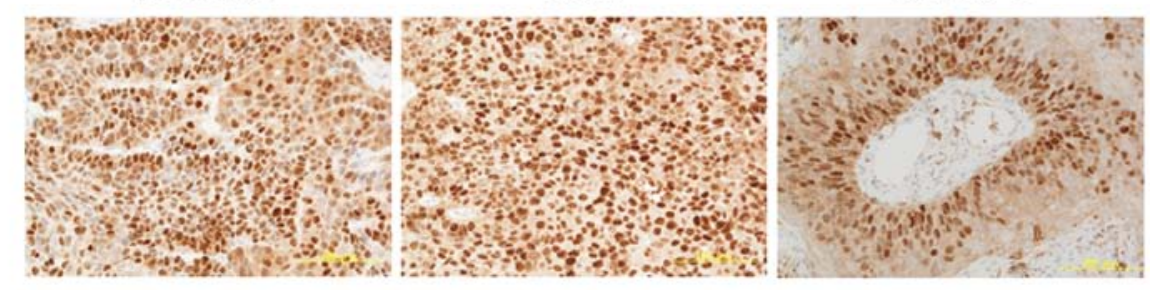

B
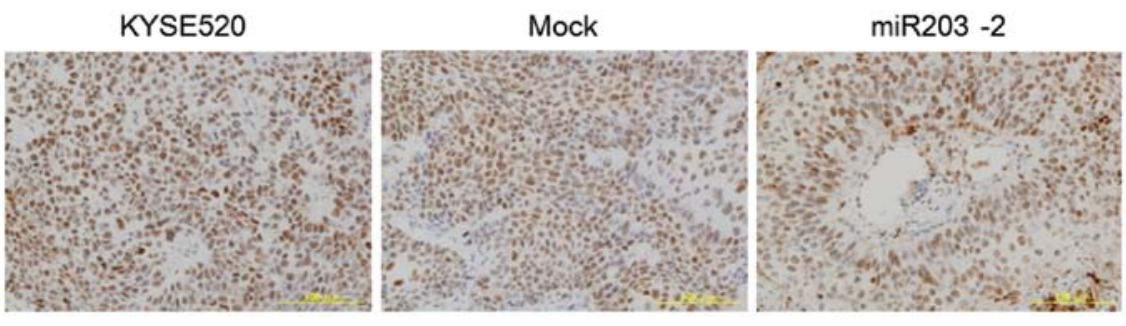

Figure 4. The expression of p63 and Bmi-1 in the miR-203 stable clone and mouse xenograft tumors. (A) Immunohistochemical staining for p63 in the mouse xenograft tumors established from KYSE520, KYSE520 mock and KYSE520 miR203-2. Original magnification, x200. (B) Immunohistochemical staining for Bmi-1 in the mouse xenograft tumors established from KYSE520, KYSE520 mock and KYSE520 miR203-2. Original magnification, x200.

were markedly upregulated in the miR-203 transfectant (Table I). Genes involved in regulating cell polarity and tissue architecture, such as Rock-1, BMP-4 and ZO-1, were also upregulated in the miR-203 transfectant (Table I).

Since p63 and Bmi-1 have been reported to be the major targets of miR-203 and to regulate stem cell properties $(7,8,17-19)$, we detected the protein expression of these molecules by immunohistochemistry. p63 expression was broadly distributed in tumors established from the parent and mock transfectant, while expression was limited in the first several layers at the center area of the foci in tumors from miR-203 stable clones (Fig. 4A). Bmi-1 was expressed in almost all cells and was broadly distributed in tumors even those from miR-203 stable clones (Fig. 4B). These results indicated that 
Table II. miR-203 expression and clinical characteristics of the patients.

\begin{tabular}{|c|c|c|c|c|}
\hline Characteristics & Total (\%) & Low $(\%)(\mathrm{n}=13)$ & $\operatorname{High}(\%)(\mathrm{n}=7)$ & P-value \\
\hline \multicolumn{5}{|l|}{ Age (48-86) } \\
\hline$<67$ & $10(50.0)$ & $5(25.0)$ & $5(25.0)$ & 0.35 \\
\hline \multicolumn{5}{|l|}{ Median 67} \\
\hline$\geq 67$ & $10(50.0)$ & $8(40.0)$ & $2(10.0)$ & \\
\hline \multicolumn{5}{|l|}{ Gender } \\
\hline Male & $17(85.0)$ & $11(55.0)$ & $6(30.0)$ & $>0.99$ \\
\hline Female & $3(15.0)$ & $2(10.0)$ & $1(5.0)$ & \\
\hline \multicolumn{5}{|l|}{ Location } \\
\hline CeUtMt & $9(45.0)$ & $6(30.0)$ & $3(15.0)$ & $>0.99$ \\
\hline LtAe & $11(55.0)$ & $7(35.0)$ & $4(20.0)$ & \\
\hline \multicolumn{5}{|l|}{ TNM } \\
\hline $\mathrm{T} 1 / \mathrm{T} 2$ & $7(35.0)$ & $4(20.0)$ & $3(15.0)$ & 0.65 \\
\hline T3/T4 & $13(65.0)$ & $9(45.0)$ & $4(20.0)$ & \\
\hline No & $5(25.0)$ & $3(15.0)$ & $2(10.0)$ & $>0.99$ \\
\hline N1 & $15(75.0)$ & $10(50.0)$ & $5(25.0)$ & \\
\hline M0 & $20(100)$ & $13(65.0)$ & $7(35.0)$ & $>0.99$ \\
\hline M1 & $0(0.0)$ & $0(0.0)$ & $0(0.0)$ & \\
\hline \multicolumn{5}{|l|}{ TNM stage } \\
\hline 2 & $8(40.0)$ & $5(25.0)$ & $3(15.0)$ & $>0.99$ \\
\hline 3 & $12(60.0)$ & $8(40.0)$ & $4(20.0)$ & \\
\hline \multicolumn{5}{|l|}{ Histology } \\
\hline Poor/Moderate & $11(55.0)$ & $8(40.0)$ & $3(15.0)$ & 0.64 \\
\hline Well & $9(45.0)$ & $5(25.0)$ & $4(20.0)$ & \\
\hline
\end{tabular}

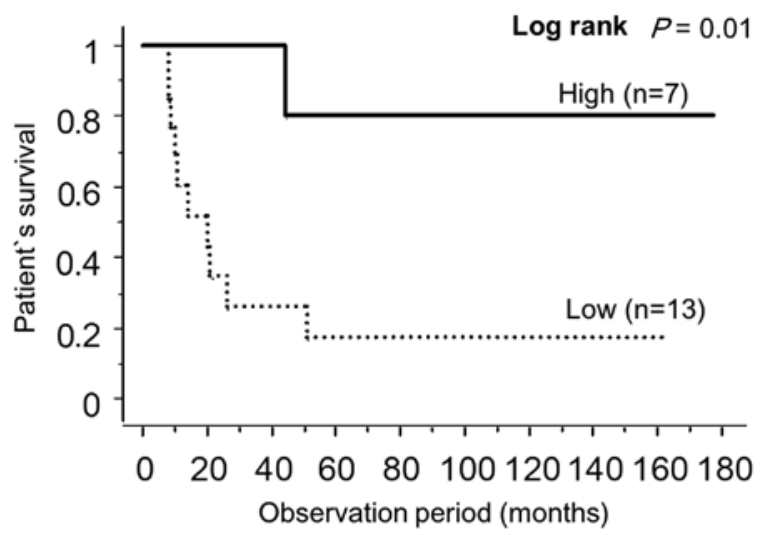

Figure 5. Correlation between miR-203 expression and favorable overall survival after curative surgery. A total of 20 ESCC patients were analyzed by Kaplan-Meier survival analysis (log-rank test).

miR203-induced suppression of p63 in supra-basal cell fraction was involved in the phenotypic change in this experiment, while Bmi-1 did not play a key role in the previous model.

miR-203 expression in ESCC specimens and clinicopathological characteristics of the patients. To assess the clinical importance of miR-203 on ESCC, we next investigated the relationship between miR-203 expression in surgically removed ESCC specimens and clinicopathological characteristics of the patients.

In the 20 specimens analyzed in this study, miR-203 expression was high in 7 specimens $(35.0 \%)$ (the relative expression level of miR-203 in tumors was $>2$-fold higher than that of normal counterparts) and low in 13 specimens (75.0\%). No significant correlation was observed between miR-203 expression and various factors such as age, gender, tumor location, extent of the tumors, lymph node metastasis, distant metastasis, pTNM pathological classification or histology (Table II). However, Kaplan-Meier survival curves revealed that miR-203 expression significantly correlated with a favorable outcome (Fig. 5). Multivariate analysis revealed that the expression of miR-203 was an independent prognostic factor $(\mathrm{P}=0.0006)$.

\section{Discussion}

Our investigation using ESCC cell lines demonstrated that the induction of miR-203 resulted in keratinocyte differentiation along with cell growth inhibition, both in vitro and in vivo. Cell growth inhibition of miR-203 in vitro has been reported in various cancers, such as lung, breast, prostate and esophagus (20-23); however, we further demonstrated significant tumor growth inhibition in a mouse xenograft model, which indicated the important role of miR-203 in cancer progression and its potential use as a target for novel therapies. In addition 
to cell growth inhibition, tumors established from the mouse xenograft experiment in our study exhibited stratified squamous differentiation with restored baso-apical tissue polarity in conjunction with production of the basement membrane, resembling normal esophageal epithelia. Our cDNA microarray analysis detected the upregulation of genes encoding basement membrane proteins (24), such as laminin and collagen type 4, and also the upregulation of genes involved in cell/tissue polarity, such as BMP-4 (25), ZO-1 (26) and Rock-1 (27), which suggested that the molecular basis of miR-203 induced the restoration of tissue architecture in our model. Based on the concept that the interaction between the basement membrane and basal cells coordinates epithelial polarity (27-29), miR-203-induced molecular processes in basal cell subset of the tumor, possibly including cancer initiating cells, was suggested to play a key role in regulating the tumor organization in vivo.

The oncogene p63 has been reported to be expressed in normal stem cells and regulates the proliferation and differentiation of keratinocytes in the epidermis and esophagus $(18,19,30)$. p63 has also been identified as a major target gene of miR-203 and its repression was shown to induce growth inhibition and differentiation in normal epidermis $(7,8,22)$. Our RT-PCR experiment with sorted cells revealed that p63 mRNA was strongly expressed in p75NTR bright cells, whereas it was downregulated in p75NTR dim cells even though miR-203 expression was equally induced in both fractions. In addition, the distribution of $\mathrm{p} 63$ protein expression detected by immunohistochemistry in xenograft tumors established from miR-203 transfectants was localized in cells residing at the basal and suprabasal layers, whereas p63 expression was diminished at the mid and outer layers along with the cell cycle exit and squamous differentiation. These results indicated that p63 suppression by miR-203 in p75NTR-dim cells induced the squamous differentiation.

Bmi-1 has been associated with stem cell self-renewal (14) and was also shown to be suppressed by miR-203, leading to a tumor suppressor effect in prostate cancer $(31,32)$, however, the expression of Bmi-1 was not suppressed in the present study. Bmi-1 expression was broadly distributed throughout mouse xenograft tumors from miR-203 transfectants, which indicated that it was not a key molecule involved in the miR-203-induced phenotypic change in our model.

Our in vitro investigation using flow cytometry revealed that the proportion of p75NTR bright cells expressing p63 and Bmil were well maintained in miR-203 transfectants despite exhibiting advanced differentiation. Taken together with reports that p75NTR is suggested to be expressed in cells with stem cell properties $(13,33,34)$, the maintained p75NTR bright cell component in our study may reflect the phenotype of the miR-203 transfectants in vivo, in which tumorigenicity was retained even though tumor growth was strongly suppressed.

In addition, based on the concept that the interaction between the basement membrane and basal cells coordinates epithelial polarity (27-29), miR-203-induced molecular processes in p75NTR-bright cells have been suggested to play a key role in regulating the architecture of tumor tissue. A more detailed investigation on the miR-203 target genes involved in the phenotypic change observed in our model may provide us with a better understanding of tumor tissue organization and also a novel target for therapy.

Our investigation on the expression of miR-203 in surgically removed ESCC specimens demonstrated that it significantly correlated with a favorable prognosis after curative surgery. No significant correlation was observed between miR-203 expression and clinical factors such as the extent of tumors, lymph node metastasis, pTNM pathological classification, and histology. However, multivariate analysis revealed that the expression of miR-203 was an independent prognostic factor, indicating that it is involved in crucial biological processes in the development and progression of ESCC.

In summary, we demonstrated that the induction of miR-203 resulted in squamous differentiation along with significant growth inhibition in vivo. Tumors established from the mouse xenograft experiment exhibited stratified squamous differentiation with restored baso-apical tissue polarity in conjunction with production of the basement membrane, resembling normal esophageal epithelia. Our cDNA microarray analysis demonstrated the upregulation of genes involved in cell polarity and tissue architecture. Investigation using surgically removed ESCC specimens further demonstrated that the expression of miR-203 significantly correlated with a favorable prognosis in patients with ESCC. These results suggest the use of miR-203 and its related molecules as novel therapeutic and diagnostic targets in patients with ESCC.

\section{Acknowledgements}

This study was supported by a Grant-in-Aid for Scientific Research (C) MEXT KAKENHI Grant no. 23591920. The authors wish to thank Ms. Sakiko Shimada, Ms. Takako Murai, and Mr. Masahiko Kawahara for cell culture and technical assistance.

\section{References}

1. Thallinger CM, Raderer M and Hejna M: Esophageal cancer: a critical evaluation of systemic second-line therapy. J Clin Oncol 29: 4709-4714, 2011.

2. Watt FM: Stem cell fate and patterning in mammalian epidermis Curr Opin Genet Dev 11: 410-417, 2001.

3. Bilder D, Li M and Perrimon N: Cooperative regulation of cell polarity and growth by Drosophila tumor suppressors. Science 289: 113-116, 2000.

4. Visvader JE and Lindeman GJ: Cancer stem cells in solid tumours: accumulating evidence and unresolved questions. Nat Rev Cancer 8: 755-768, 2008.

5. Ginestier C, Wicinski J, Cervera N, Monville F, Finetti P, Bertucci $\mathrm{F}$, et al: Retinoid signaling regulates breast cancer stem cell differentiation. Cell Cycle 8: 3297-3302, 2009.

6. Baek D, Villén J, Shin C, Camargo FD, Gygi SP and Bartel DP: The impact of microRNAs on protein output. Nature 455: 464-471, 2008.

7. Lena AM, Shalom-Feuerstein R, Rivetti di Val Cervo P, Aberdam D, Knight RA, Melino G and Candi E: miR-203 represses 'stemness' by repressing DeltaNp63. Cell Death Differ 15: $1187-1195,2008$

8. Yi R, Poy MN, Stoffel M and Fuchs E: A skin microRNA promotes differentiation by repressing 'stemness'. Nature 452: 225-229, 2008.

9. Okumura T, Tsunoda S, Mori Y, Ito T, Kikuchi K, Wang TC, et al: The biological role of the low-affinity p75 neurotrophin receptor in esophageal squamous cell carcinoma. Clin Cancer Res 12: 5096-5103, 2006.

10. Shimada $\mathrm{Y}$, Imamura $\mathrm{M}$, Wagata $\mathrm{T}$, Yamaguchi $\mathrm{N}$ and Tobe $\mathrm{T}$ : Characterization of 21 newly established esophageal cancer cell lines. Cancer 69: 277-284, 1992. 
11. Sobin LH, Gospodarowicz M and Wittekind C (eds): TNM Classification of Malignant Tumours. UICC International Union Against Cancer 2010. 7th edition. Wiley-Blackwell, Hoboken, NJ, 2010.

12. Osawa S, Shimada Y, Sekine S, Okumura T, Nagata T, Fukuoka J and Tsukada K: MicroRNA profiling of gastric cancer patients from formalin-fixed paraffin-embedded samples. Oncol Lett 2 613-619, 2011.

13. Okumura T, Shimada Y, Imamura M and Yasumoto S: Neurotrophin receptor $\mathrm{p} 75$ (NTR) characterizes human esophageal keratinocyte stem cells in vitro. Oncogene 22: 4017-4026, 2003.

14. Liu S, Dontu G, Mantle ID, Patel S, Ahn NS, Jackson KW, Suri P and Wicha MS: Hedgehog signaling and Bmi-1 regulate selfrenewal of normal and malignant human mammary stem cells. Cancer Res 66: 6063-6071, 2006.

15. Hosen N, Yamane T, Muijtjens M, Pham K, Clarke MF and Weissman IL: Bmi-1-green fluorescent protein-knock-in mice reveal the dynamic regulation of bmi-1 expression in normal and leukemic hematopoietic cells. Stem Cells 25: 1635-1644, 2007.

16. Koster MI, Kim S, Mills AA, DeMayo FJ and Roop DR: p63 is the molecular switch for initiation of an epithelial stratification program. Genes Dev 18: 126-131, 2004

17. Jin J, Deng J, Wang F, Xia X, Qiu T, Lu W, Li X, Zhang $\mathrm{H}, \mathrm{Gu} \mathrm{X}, \mathrm{Liu} \mathrm{Y}, \mathrm{Cao} \mathrm{W}$ and Shao W: The expression and function of microRNA-203 in lung cancer. Tumour Biol 34: 349-357, 2013

18. Daniely Y, Liao G, Dixon D, Linnoila RI, Lori A, Randell SH, et al: Critical role of p63 in the development of a normal esophageal and tracheobronchial epithelium. Am J Physiol Cell Physiol 287: C171-C181, 2004

19. Truong AB, Kretz M, Ridky TW, Kimmel R and Khavari PA: p63 regulates proliferation and differentiation of developmentally mature keratinocytes. Genes Dev 20: 3185-3197, 2006.

20. Wang C, Zheng X, Shen C and Shi Y: MicroRNA-203 suppresses cell proliferation and migration by targeting BIRC5 and LASP1 in human triple-negative breast cancer cells. J Exp Clin Cancer Res 31: 58, 2012.

21. Viticchiè G, Lena AM, Latina A, Formosa A, Gregersen LH Lund AH, et al: MiR-203 controls proliferation, migration and invasive potential of prostate cancer cell lines. Cell Cycle 10: 1121-1131, 2011.

22. Yuan Y, Zeng ZY, Liu XH, Gong DJ, Tao J, Cheng HZ and Huang SD: MicroRNA-203 inhibits cell proliferation by repressing $\triangle \mathrm{Np} 63$ expression in human esophageal squamous cell carcinoma. BMC Cancer 11: 57, 2011.
23. Takeshita N, Mori M, Kano M, Hoshino I, Akutsu Y, Hanari N, et al: miR-203 inhibits the migration and invasion of esophageal squamous cell carcinoma by regulating LASP1. Int J Oncol 41: 1653-1661, 2012

24. Yurchenco PD: Basement membranes: cell scaffoldings and signaling platforms. Cold Spring Harb Perspect Biol 3: pii: a004911, 2011.

25. Yoshida S, Yasuda M, Miyashita H, Ogawa Y, Yoshida T, Matsuzaki Y, et al: Generation of stratified squamous epithelial progenitor cells from mouse induced pluripotent stem cells. PLoS One 6: e28856, 2011.

26. Nagaoka K, Udagawa T and Richter JD: CPEB-mediated ZO-1 mRNA localization is required for epithelial tight-junction assembly and cell polarity. Nat Commun 3: 675, 2012.

27. Daley WP, Gervais EM, Centanni SW, Gulfo KM, Nelson DA and Larsen M: ROCK1-directed basement membrane positioning coordinates epithelial tissue polarity. Development 139: 411-422, 2012.

28. Zen K, Yasui K, Gen Y, Dohi O, Wakabayashi N, Mitsufuji S, et al: Defective expression of polarity protein PAR-3 gene (PARD3) in esophageal squamous cell carcinoma. Oncogene 28: 2910-2918, 2009.

29. Bryant DM and Mostov KE: From cells to organs: building polarized tissue. Nat Rev Mol Cell Biol 9: 887-901, 2008.

30. Pellegrini G, Dellambra E, Golisano O, Martinelli E, Fantozzi I, Bondanza S, et al: p63 identifies keratinocyte stem cells. Proc Natl Acad Sci USA 98: 3156-3161, 2001.

31. Saini S, Majid S, Yamamura S, Tabatabai L, Suh SO, Shahryari V, et al: Regulatory role of miR-203 in prostate cancer progression and metastasis. Clin Cancer Res 17: 5287-5298, 2011.

32. Boll K, Reiche K, Kasack K, Mörbt N, Kretzschmar AK, Tomm JM, et al: MiR-130a, miR-203 and miR-205 jointly repress key oncogenic pathways and are downregulated in prostate carcinoma. Oncogene 32: 277-285, 2012.

33. Nakamura T, Endo K and Kinoshita S: Identification of human oral keratinocyte stem/progenitor cells by neurotrophin receptor p75 and the role of neurotrophin/p75 signaling. Stem Cells 25: 628-638, 2007.

34. Huang SD, Yuan Y, Liu XH, Gong DJ, Bai CG, Wang F, et al: Self-renewal and chemotherapy resistance of p75NTR positive cells in esophageal squamous cell carcinomas. BMC Cancer 9: 9, 2009. 